cytatów, uzupełnień itp. lepiej w ogóle nie wspominać, chyba że na seminariach edytorsko-tekstologicznych lub naukach pomocniczych, gdzie praca Wejs-Milewskiej może być wyrazistym przykładem na to, jak książek wydawać nie należy.

Podsumowując, trzeba powtórzyć stwierdzenie początkowe, głoszące iż praca Radio Wolna Europa na emigracyjnych szlakach pisarzy jest pozycją interesującą i pożyteczną, a w warstwie przywoływania nieznanych szerzej źródeł zgoła nie do przecenienia. W sferze badawczej dysertacja z powodzeniem realizuje zasygnalizowany przez autorkę postulat poszerzania stanu istniejącej wiedzy przy jednoczesnym zachowaniu dotychczasowych ocen dorobku poszczególnych pisarzy. Co do samej metody formułowania, a następnie dowodzenia stawianych tez, to - w odczuciu piszącego te słowa - książka pozostawia szerokie pole do dyskusji, a niektóre uwagi czynione przez autorkę wydają się niesłuszne. Natomiast w zakresie dokumentowania faktów historycznych, literackich i radiowych praca w widoczny sposób niedomaga, zaś jej strona wydawniczo-redakcyjna jest absolutnie naganna.

Rafał Moczkodan (Toruń)

\title{
Displaced Persons
}

Christian und Marianne Pletzing (Hgg.), Displaced Persons. Flüchtlinge aus den baltischen Staaten in Deutschland. München: Martin Meidenbauer Verlagsbuchhandlung, 2007, 246 s., il.

Der Band 12 der Reihe Colloquia Baltica, den Marianne und Christian Pletzing herausgegeben haben, geht aus ein Seminar zurück, das die Academia Baltica in Lübeck mit der Gustav-Heinemann-Bildungsstätte in Malente unter dem Patronat des Honorarkonsulats der Republik Lettland in Schleswig-Holstein veranstaltet haben.

Vaira Vikse-Freiberga, bis Juli 2007 die Staatspräsidentin der Republik Lettland geleitet mit ihrem kurzen Text in die Thematik, in dem sie freilich die lettischen DPs herausstellt, gleichwohl beziehen sich ihre Behauptungen allgemein auf die Balten: „Im Exil ließen die Balten die Köpfe nicht hängen“, und in diesem Sinne erweitert die Verf. ihre Perspektive als sie zurecht feststellt:

Die baltischen Displaced Persons sind nicht nur ein Teil der lettischen, litauischen und estnischen Geschichte, sondern in gewisser Weise auch Bestandteil der deutschen Regionalgeschichte (S. 7-8).

Das Vorwort stammt von Feder Christian Pletzings, des Leiters der Academia Baltica in Lübeck. Er präsentiert die wenig bekannten Aspekte der Nachkriegszeit im historischen Bewusstsein von heute. Dabei stützt er sich auf die Pionierarbeit und auch das bisher einzige deutschsprachige Standardwerk zu den DPs im besetzten Deutschland von Jacobmeyer. Der Autor bemängelt richtig, dass vor allem die zahlreichen Erfahrungsberichte baltischer DPs aus den USA, Kanada und Australien in der Forschung kaum rezipiert worden seien. Folgerichtig verweist er mit Nachdruck auf die am Ende der Publikation abgedruckten Zeitzeugenberichte (S. 201-221). Den folgenden Beiträgen vorweg nimmt Pletzing summierend, dass es gerade der baltischen Gruppe der DPs eigen war, sich insbesondere aus Intellektuellen und Angehörigen des Bildungsbürgertums zusammenzusetzen. 
Tillmann Tegeler (Osteuropa-Institut München — heute [2008] Regensburg) befasste sich mit dem Thema: „Esten, Letten und Litauer in Nachkriegsdeutschland. Von rechtlosen Flüchtlingen zu heimatlosen Ausländern“. Er bietet damit einerseits einen Überblick über das Thema der DPs, die in der Bundesrepublik Deutschland seit 1951 als heimatlose Ausländer bezeichnet werden; auf der anderen Seite bekräftigt Tegeler die allgemein geltende Zäsur des Jahres 1951, in dem die Geschichte der DPs in Westdeutschland endet. Der Verf. betrachtet die DPs als einen Teil der gemeinsamen alliierten Geschichte nach 1945. Speziell zum Baltikum skizziert er die verschiedenen Flüchtlingswellen und -gruppen, die die Opfer der politischen Entwicklung von 1939 bis 1945 umfassen. Für die Nachkriegzeit betont er die Entstehung geheimer Lager für die so genannten disputed persons, also für die Balten, die des Antistalinismus wegen nicht in ihre Heimat zurückkehren wollten, jedoch gemäß der Verträge von Jalta und Halle zwischen den Westalliierten und der Sowjetunion zur Zwangsrepatriierung hätten gezwungen werden können. Überdies wurde der Alltag der Balten angesprochen, ihre Beteiligung an der „DP-Studentenbewegung“ innerhalb der Universitäten der United Nations Relief and Rehabilitation Administration, der UNRRA-Universität in München, sowie der Baltischen DP-Universität in Hamburg/Pinneberg. Zum sozialpolitischen Alltag gehörte die Tätigkeit der Nationalen Komitees der Esten, Letten und Litauer in Deutschland der unmittelbaren Nachkriegszeit genauso, wie die des jeweiligen Roten Kreuzes. Explizit geht Tegeler auf das Resettlement ein — die bekannten britischen Einsiedlungsoperationen Westward-Ho und Balt-Cygnet - und stelle fest, dass diese Pläne nicht nur eine Reaktion auf die gescheiterte Arbeit der UNRRA gewesen sei, sondern dass die Westalliierten bereits während des Zweiten Weltkrieges in den entwurzelten Menschen Europas, zu mal auf dem Gebiet des „Dritten Reiches“, für sich ein zukünftiges Arbeitskräftepotenzial gesehen hätten. So sind die Pläne des Resettlement jedenfalls auf die Zeit vor 1946/1947 zu datieren (S. 13-27).

Dorothee M. Goeze (Herder-Institut Marburg) stellt in ihrem Beitrag „Alltag estischer DPs in Deutschland. Die Sammlung Karl Nikolei Hintzer im Herder-Institut Marburg“ zahlreiche der insgesamt 24.000 Bilder des estischen Bildberichterstatters Karl Nikolai Hintzer vor. Ihre Einführung veranschaulicht in erster Linie das Alltagsleben der in die westlichen Besatzungszonen geflohenen Esten in den DPLagern bis in die 1950er-Jahre. Den visuellen Beitrag — insgesamt 48 Bilder — teilt die Autorin in mehrere Unterthemen ein: Organisationen der DPs, Versorgung und Hilfsprogramme - interne, wie die Schaffung der Lager-Apotheken, und externe, wie die der UNRRA, der International Refugee Organization (IRO) und des Lutherischen Weltbundes —, ferner Ausbildung — hier insbesondere die Pinneberger Universität —, des Weiteren Beschäftigung - welche der Bildautor als Zwangsarbeit bezeichnet hatte —, sowie Wachkompanien des Labor Services der US-Army, Altenheime, Sport, Kinder, Kultur, Kirche und nationale Feiertage. Summierend verwendet die Verf. den Begriff „Klein-Estland“, das die DPs in den Lagern in und um Lübeck erstehen ließen, und das Karl Hintzer in der Photographie verewigte (S. 29-61).

Stefan Schröder (Stadtarchiv Greven) widmet sich dem Komplex „Nachbarschaft und Konflikt. Die DPs und die Deutschen“. Zunächst kritisiert Schröder den Forschungsfortschritt zum Thema der DPs, nicht nur der baltischen, sondern im Allgemeinen. Als Gegenpol diente das Thema der Zwangsarbeit, das, weil politisch gewollt, eine verbreitete Rezeption in der Wissenschaft und Publizistik erfahren hatte. Leider ging der Funke nicht auf die Erforschung der anschließenden Epoche über. Indem Schröder an die Rassenskala für die Völker und Nationen im Nationalsozialismus erinnert, geht er zum eigentlichen Thema des Aufsatzes über und 
erläutert die Stellung der Balten in diesem Un-,,Rechtssystem“, um in die Nachkriegsgeschichte zu wechseln. Diese Perspektive erscheint hilfreich, um 1945 aufgetretene Phänomene der Brutalität und Rechtlosigkeit gegenüber der deutschen Bevölkerung zu erklären; die Übrigen Phänomene, die nur sehr kurzlebig waren, sich jedoch in die kollektive Erinnerung eingeschrieben haben und dabei nicht zuletzt die NS-Propaganda rezipierten. Bei der allgemeinen Ablehnung der DPs wies die deutsche Gesellschaft dennoch Vorlieben auf, von welchen insbesondere die Balten profitierten, da sie allgemein als arbeitsam, tüchtig und den Deutschen nahe stehend empfunden wurden. Was bisher unerforscht bleibt, so der Autor, sei der Alltag, der wichtiger als alle Vorurteile war, in einer Zeit des Überlebens und Improvisierens. Hervorgehoben wird der Faktor Lager, charakteristisch für die Nachkriegszeit für mehrere Gruppen von Flüchtlingen. Überdies wird die deutsche Sprache behandelt, die in den mehrsprachigen Lagern zum einzig möglichen und damit willkommenen Mittel der Kommunikation wurde. Sport erwies sich als besonders effizienter Weg für die DPs, um mit Deutschen in Kontakt zu treten. Trotz aller positiv und negativ empfundenen Berührungspunkte auf beiden Seiten, hingen die Beziehungen zu den Deutschen von der jeweiligen Person ab, ihrer Vorstellungen und Absichten, in Deutschland zu bleiben oder auszuwandern, und eventuell den Hoffnungen, einst wieder in die Heimat zurückkehren zu können. Abschließend erklärt Schröder, warum es nicht möglich sei, die Beziehungsgeschichte zwischen DPs und Deutschen aus der Aktenüberlieferung der Behörden und den Statistiken zu schreiben und hebt die Methode der oral history für die Aufarbeitung dieses Themas hervor (S. 63-83).

Christian Pletzing (Academia Baltica Lübeck) widmet sich der „Stadt der Displaced Persons” und beschreibt die „DPs aus den baltischen Staaten in Lübeck“. Hierfür verwendet die Verf. das Schrifttum aus dem Stadtarchiv der Hansestadt, die zeitgenössische Presse, zahlreiche Quellen aus privaten Nachlässen und estnische sowie deutsche Fachliteratur. Einleitend erweitert Pletzing die historische Perspektive und zeigt alle Gruppen im wenige zerstörten, doch sehr überfüllten Lübeck: die Einheimischen, deutsche Flüchtlinge und Vertriebene und eben Ausländer. Aus dieser Konstellation heraus ergaben sich zahlreiche Konflikte, die geschildert werden. Die Kriminalität - die keineswegs allein für die DPs charakteristisch war, zumal der sozialen Probleme der Nachkriegszeit - diskutiert Pletzing, indem er auf Statistiken verweist. Darin sind die meisten Delikte von Polen und nicht von Balten verübt worden, was sich mit der sozialen Zusammensetzung und der Sozialisation der DPs als Zwangsarbeiter im „Dritten Reich“ erklären lässt. Ferner beschreibt der Autor das kulturelle Leben der DPs, die Institutionen und die — in diesem Zusammenhang — die DP-Presse. Der Beitrag endet mit der Schilderung des Status eines Heimatlosen Ausländers und des schwierigen Alltags der DPs, insbesondere bis zum sog. Wirtschaftswunder (S. 85-106).

Svetlana Červonnaja (Universität Toruń/Polen) gibt einen Überblick zu den „Litauern in Deutschland nach dem Zweiten Weltkrieg. Zur Situation der litauischen Kultur im Exil“. Zunächst bemängelt sie, dass bisher nur die litauische Exilliteratur in den USA untersucht, die in Nachkriegsdeutschland erschienene hingegen vernachlässigt worden sei. In der UdSSR und in der GUS fand und findet die Rezeption dieser Literatur kaum statt, trotz der Verantwortung dieses Landes für das Exil der Litauer. Die Autorin stellt neun Flucht- oder Auswanderungswellen aus Litauen seit 1939 fest — somit neun Epochen des künstlerischen Schaffens im Exil —, zuletzt erwähnt sie die Arbeitsemigration nach 1990. Für die Künstler und alle Litauer in Deutschland waren die Jahre 1945 bis 1949/1950 von besonderer Bedeutung. Denn die litauischen DPs waren in ihrer Mehrheit keine ehemaligen Zwangsarbeiter, sondern 
Flüchtlinge; und geflüchtet waren insbesondere Intellektuelle, Wissenschaftler und Politiker, alle jene Personen also, die Angst hatten, von der Sowjetmacht unterdrückt zu werden. Diese ,intellektuelle Armee“ stellte immerhin zehn Prozent aller litauischen DPs dar, in der Heimat verblieb die Intelligenz lediglich mit einem Anteil von einem Prozent. Über die Hervorhebung des litauischen Kulturzentrums in Wien seit August 1944, und der Schilderung des Vorfalls in Schweden, von wo litauische DPs noch 1946 an die UdSSR ausgeliefert worden waren, geht Červonnaja zum Kern des Themas über, zu den einzelnen Künstlern: Bildhauer, Holzschneider, Maler, Grafiker, Architekten und Schriftsteller. Dabei fielen Begriffe und Probleme wie politische Kunst, politische Opposition in Litauen — die Waldbrüder — oder sowjetische Infiltration des Exils. Ferner nennt die Verf. einige politische Institutionen der Exil-Litauer, an der Spitze den Verband der Litauer im Exil, der schon im November 1945 in Wiesbaden eine Konferenz abgehalten hatte. Sie erwähnt auch Kultur-Institutionen des litauischen Exils, wie beispielsweise das Institut für Angewandte Kunst, das mit Hilfe Frankreichs in der französischen Besatzungszone entstanden war und gearbeitet hatte. Diese Einrichtung diente allen Nationalitäten, gleichwohl stellten Litauer 80 Prozent der Absolventen dieser Bildungsanstalt dar. Den politischen Beitrag der Schriftsteller, beispielsweise in der Zusammenarbeit mit dem Radio Free Europe in München, wurde ebenfalls kurz angesprochen. Interessant ist die Erweiterung des Themas über Kulturbeziehungen zwischen „Deutschland“ und Litauen um die ehemalige DDR; die Autorin verweist dabei auf die von der DDR aus ermöglichten Kontakte zwischen Künstlern in der sowjetischen Republik Litauen und den Ostdeutschen. Abschließend wird mit Hilfe Vincas Bartusevičius festgestellt, dass nach der Wiedererlangung der Unabhängigkeit durch Litauen die litauischen Flüchtlinge des Jahres 1944 und deren Nachfahren von der Pflicht entlassen werden, Auslandslitauer zu sein; sie sind frei, da ihre Heimat auch frei ist (107-138).

Ingo Hoddick (Musikjournalist Köln/Duisburg) widmet sich dem Thema „Musiker als Displaced Persons. Vladas Jakubénas und Jāzeps Vītols in Deutschland“. Hoddick präsentierte beider Künstler, den Letten Vitols und den Litauer Jakubenas, setzt sich jedoch besonders mit Jakubenas auseinander. Außer Musik und deren fachmännischen zeitlichen und künstlerischen Einordnung wurden die Viten beider Musiker erzählt. Vitols war auch im Deutschland der Vorkriegszeit bekannt, da er zahlreiche Artikel für Musikzeitschriften verfasst hatte. Jakubenas komponierte in den DP-Lagern ununterbrochen, da er dort — wie er selbst sagte — Zeit und Publikum gehabt habe. 1949 wanderte er in die USA aus und verließ sie bis zu seinem Tode nicht mehr. Der Autor erwähnt zuletzt die Bedeutung der Exilmusik für die Heimatländer sowie die damit zusammenhängenden Umbettungen von deren Gebeinen in die Heimat, welche Rückkehr aus dem Exil nicht nur Jakubénas, sondern unter anderem auch der Musiker Kacynskas posthum erlebten. Zahlreiche Künstler werden erwähnt und kurz dargestellt; insgesamt ein kurzer Beitrag mit unglaublicher Fülle an fachmännischer Information (S. 139-148).

Anderas Fülberth (Universität Kiel) stellt „Den lettischen Schriftsteller Jānis Janusudrabiņš und die Stationen seines Exils in Westfalen. Eine Betrachtung unter regionalgeschichtlichen Aspekten“ vor. Der Künstler, bedeutender Maler, jedoch insbesondere auch Dichter und Schriftsteller, war in der Zwischenkriegszeit der meist gelesene Schriftsteller Lettlands. Im Exil lebte er von 1945 bis 1948 in Bielefeld, dann bis zum Tode in Körbecke am Möhnesee. Das literarische Werk Janusudrabiņš präsentiert der Autor in historischen Zusammenhängen des Ersten Weltkrieges, der Oktoberrevolution, des Zweiten Weltkrieges und des Exils. Selbstverständlich spiegelt sich darin auch die Geschichte des Baltikums mit seinen Okkupationsperioden von 1939 
bis 1991 wieder. Den Anstoß, ins Exil zu gehen, gab Janusudrabiṇš der 'Soziozid' im Baltikum während der ersten sowjetischen Okkupation 1940 bis 1941. Zum deutschen Besatzer hatte der Künstler überhaupt keine Beziehung, nicht deshalb also floh er ins zum Scheitern verurteilte „Dritte Reich“. Nach einem sehr schwierigen Anfang, zeitweise in einem DP-Lager, halfen Janusudrabiņš familiäre Beziehungen, sich in der britischen Besatzungszone in Körbecke bescheiden einzurichten und künstlerisch wieder aktiv zu werden. Seine Werke sind nur in einigen — nicht ganz gelungenen - Übersetzungen bekannt. Da er als Schriftsteller 1948 in Stockholm geehrt wurde, am Möhnsee als Künstler einen gewissen Bekanntschaftsstatus erlangte, nahm er an Dichtertreffen teil, die vom Landschaftsverband Nordrhein-Westfallen veranstaltet wurden. Dabei spielte er eine wichtige Rolle 1955, als er erfolgreich um Versöhnung innerhalb des deutschen Künstlerkreises bemüht war. Janusudrabiņš starb in Deutschland 1962, im Jahre 1997 kehrten seine Gebeine nach Lettland zurück (S. 149-164).

Peter Wörster (Herder-Institut Marburg) stellt „Das Estnische Zonenarchiv. Möglichkeiten und Grenzen der Erforschung des DP-Schicksals“. Marburg ist ein besonderer Ort, an dem zahlreiche sog. Ost-Archivalien aufbewahrt werden. Hierzu zählt auch „Das Estnische Zonenarchiv Marburg“, angeregt von Hellmuth Weis, der 1951 die Bibliothek des Herder-Instituts mit aufbaute. Das bundesweite Estnische Zentralkomitee in Ulm bat gerade dieses Institut - mit Unterstützung der späteren Balitischen Historischen Kommission - , das Estnische Zonenarchiv in Depositum zu nehmen, so die Genese dieses Bestandes, bevor dieses Deutschland in die USA verließ. Wörster geht entsprechend der begrenzen Einsicht auch auf die Inhalte dieser Überlieferung ein, um schließlich darzulegen, was die Forschung noch leisten könnte, wenn die Quellen, die nun in Lakewood / USA einst ausgewertet würden (S. 165-174).

Daina Zalāne (DP-Historikerin, Riga) präsentiert das Internet-Projekt „'DP Album'. Das Leben lettischer DPs in Bildern“" vor. Das Portal www.dpalbums.lv bietet gegenwärtig ungefähr 2000 Bilder, jeweils mit einer kurzen Beschreibung des Inhaltes. Zudem werden stets Autor/Inhaber des Bildes sowie Ort und Zeit der Aufnahme genannt. Die Internetseite verfügt über eine Struktur, die sich nach Besatzungszonen und Ortschaften orientiert, alternativ kann nach den Bildautoren/-inhabern gebrowst oder gezielt nach bestimmten Themen ortsübergreifend gesucht werden, beispielsweise Politik, kirchliches Leben, Auswanderung, Versorgung etc. Neben der Bild-Datenbank bieten die Autoren des Portals eine kleine Internetausstellung in Lettisch, Englisch und Deutsch. Diese Ausstellung zeigt ausgewählte Fotos aus der zugänglichen Datenbank mit einer Beschreibung der zeithistorischen Zusammenhänge. Als solche ist diese Ausstellung für das Thema der DPs allgemein gültig und nicht nur für die der lettischen DPs. Zalānes beschreibt, wie DPs in der Heimat wahrgenommen werden, dass dieses Thema innenpolitisch, gerade deshalb relevant ist, weil die letzte Präsidentin des Landes eine in Lübeck aufgewachsene DP war. Den Kern dieses Beitrages stellen 28 Bilder dar, die entsprechend der geschilderten Online-Datenbank dargestellt sind (175-198).

Zuletzt finden sich im Band drei Zeitzeugenberichte, eine englische Zusammenfassung und Informationen zu den Atoren. Es feht auch nicht an einem Ortsregister.

Insgesamt ist der Band deshalb zu würdigen, da er einerseits bezüglich der baltischen DPs einmalig viele Informationen bietet, auf der anderen Seite die im Band selbst bemängelte Rezeption des Themas in der Geschichtswissenschaft zumindest etwas füllt.

Roman P. Smolorz 